\title{
A NOTE ON A THEOREM OF J. DIESTEL AND B. FAIRES
}

\author{
J. C. FERRANDO AND M. LÓPEZ-PELLICER
}

(Communicated by Paul S. Murphy)

Dedicated to Professor M. Valdivia on the occasion of his sixtieth birthday

\begin{abstract}
Applying a property concerning certain coverings of $l_{0}^{\infty}(X, \mathscr{A})$ that always contain some elements that are barrelled and dense in $l_{0}^{\infty}(X, \mathscr{A})$, we generalize a localization theorem of $\mathrm{M}$. Valdivia, relative to vector bounded finitely additive measures (Theorem 1), and obtain two different generalizations of a theorem of J. Diestel and B. Faires ensuring that certain finitely additive measures are countably additive (Theorems 2 and 3 ).

The original proof of the quoted theorem of Diestel and Faires uses a theorem of Rosenthal that is not required in our proof of Theorem 3. This avoids imposing over the Valdivia's $\Lambda_{r}$-spaces defining the measure range space, the condition that they do not contain a copy of $l^{\infty}$.
\end{abstract}

\section{INTRODUCTION}

From now onwards the word "space" will mean "locally convex Hausdorff space over the field $K$ of the real or complex numbers." We set $\mathscr{A}$ to denote a $\sigma$-algebra of subsets of a set $X$ and represent by $e(A)$ the characteristic function of the subset $A$ of $X$. Let $l_{0}^{\infty}(X, \mathscr{A})$ be the linear space generated by the family $\{e(A), A \in \mathscr{A}\}$ endowed with the topology defined by the supremum norm. As usual, we shall identify the space $\mathbf{B}(\mathscr{A})$ of the bounded finitely additive scalar measures on $\mathscr{A}$ with the topological dual of the space $l_{0}^{\infty}(X, \mathscr{A})$, and the subspace of the countably additive scalar measures will be denoted by $\mathbf{M}(\mathscr{A})$.

A space $E$ is dual locally complete [6] if $E^{\prime}\left(\sigma\left(E^{\prime}, E\right)\right)$ is locally complete. A space $E$ is $\Gamma_{r}$ [8] $\left(\Lambda_{r}\right.$, [6]) if given any quasi-complete (locally complete) subspace $G$ of $E^{*}\left(\sigma\left(E^{*}, E\right)\right)$ such that $G$ meets $E^{\prime}$ in a dense subspace of $E^{\prime}\left(\sigma\left(E^{\prime}, E\right)\right), G$ contains $E^{\prime}$. $B_{r}$-complete spaces are $\Gamma_{r}$, and reflexive Banach spaces and Fréchet-Schwartz spaces provide some simple examples of $\Lambda_{r}$-spaces. For simplicity we introduce the following definition.

Definition. Given any positive integer $p$, a countable family of subspaces $W=$ $\left\{L_{m_{1} m_{2} \ldots m_{s}}, m_{r} \in \mathbb{N}, 1 \leq r \leq s \leq p\right\}$ of a linear space $L$ is a $p$-net in

Received by the editors September 17, 1990 and, in revised form, February 4, 1991. 1991 Mathematics Subject Classification. Primary 46A07, 46G10.

Key words and phrases. Closed graph theorems, dual locally complete spaces, $\Gamma_{r}$ and $\Lambda_{r}$-spaces, barrelled spaces, finitely (countably) additive vector measure, bounded vector measure.

The second author was partially supported by DGICYT, PS88-0050, and PS88-0114. 
$L$ if the sequence $\left\{L_{m_{1}}, m_{1} \in \mathbb{N}\right\}$ is increasing and covers $L$ and for each $s \in\{2, \ldots, p\},\left\{L_{m_{1} \ldots m_{s-1} m_{s}}, m_{s} \in \mathbb{N}\right\}$ is increasing and covers $L_{m_{1} m_{2} \ldots m_{s-1}}$.

We shall denote by $W_{p}$ the family $\left\{L_{m_{1} m_{2} \ldots m_{p}}, m_{i} \in \mathbb{N}, 1 \leq i \leq p\right\}$. In [3, Theorem 1] we have shown that if $W$ is a $p$-net in $l_{0}^{\infty}(X, \mathscr{A})$, then there exists some $L_{m_{1} m_{2} \ldots m_{p}}$ that is a dense and barrelled subspace of $l_{0}^{\infty}(X, \mathscr{A})$. This result for $p=1$ has been obtained by M. Valdivia in [7, Theorem 1] showing that $l_{0}^{\infty}(X, \mathscr{A})$ is suprabarrelled.

From the suprabarrelledness of $l_{0}^{\infty}(X, \mathscr{A})$, the following two results have been derived.

(a) Let $\mu$ be a bounded additive measure from a $\sigma$-algebra $\mathscr{A}$ on $X$ into a space $E$. Let $\left\{F_{n}, n=1,2, \ldots\right\}$ be an increasing sequence of $\Gamma_{r}$-spaces covering a space $F$. If $f: E \rightarrow F$ is a linear mapping with closed graph, there is a positive integer $q$ such that $f \mu$ is a $F_{q}$-valued bounded finite additive measure on $\mathscr{A}$ [7, Theorem 4].

(b) Let $\mu$ be a finitely additive measure on $\mathscr{A}$ with values in $E$, and let $H$ be a $\sigma\left(E^{\prime}, E\right)$ total subset of $E^{\prime}$ such that $u \mu$ is a countably additive measure for each $u \in H$. If $E$ is a countable inductive limit of $B_{r}$-complete spaces that do not contain $l^{\infty}$, then $\mu$ is a countable additive measure [5, 9.4, p. 367].

This last result extends a well-known theorem of J. Diestel and B. Faires [1, Theorem 1.1].

Our previously quoted result of [3] enables us to generalize results (a) and (b) in Theorems 1 and 2 below. Besides, Theorem 2 has suggested to us a new generalization of the Diestel-Faires theorem, avoiding the condition that the range spaces do not contain a copy of $l^{\infty}$.

\section{RESULTS}

Theorem 1. Let $\mu$ be a bounded additive measure on $\mathscr{A}$ with values in a space $E$. Suppose that $F$ is a space with a p-net $W$ such that each $L \in W_{p}$ has a locally convex topology $\mathscr{T}_{L}$ stronger than that induced by $F$, under which $L\left(\mathscr{T}_{L}\right)$ is a $\Gamma_{r}$-space. If $f$ is a linear mapping from $E$ into $F$ with closed graph, then there exists a $G \in W_{p}$ such that $f \mu$ is a $G\left(\mathscr{T}_{G}\right)$-valued bounded finitely additive measure.

Proof. As $\mu$ is bounded, the mapping $S: l_{0}^{\infty}(X, \mathscr{A}) \rightarrow E$, such that $S(e(A))=$ $\mu(A)$ for every $A \in \mathscr{A}$, is continuous, and therefore the linear map $T=f S$ has closed graph. By [3, Theorem 1], there is some $G \in W_{p}$ such that $H=T^{-1}(G)$ is dense in $l_{0}^{\infty}(X, \mathscr{A})$ and barrelled.

According to Theorems 1 and 14 of [8], the restriction of $T$ to $H$ admits a continuous extension $U$ in $l_{0}^{\infty}(X, \mathscr{A})$ with values in $G$. As $T$ has closed graph, $T=U$.

Theorem 2. Let $\mu$ be a finitely additive measure on $\mathscr{A}$ with values in a space $E$, and let $H$ be a $\sigma\left(E^{\prime}, E\right)$-total subset of $E^{\prime}$. Suppose that $E$ has a p-net $W$ such that in each $L \in W_{p}$ there exists a locally convex topology $\mathscr{T}_{L}$ finer than that induced by $E$, under which $L\left(\mathscr{T}_{L}\right)$ is a sequentially complete $\Gamma_{r}$-space not containing any copy of $l^{\infty}$. If $u \mu$ is a countably additive measure for each $u \in H$, then there exists $a G \in W_{p}$ such that $\mu$ is a $G\left(\mathscr{T}_{G}\right)$-valued countably additive vector measure. 
Proof. Let $F$ denote the linear hull of $H$. The mapping $S$ from $l_{0}^{\infty}(X, \mathscr{A})$ into $E$, such that $S(e(A))=\mu(A)$ for every $A \in \mathscr{A}$, has closed graph, since by hypothesis $u \mu \in \mathbf{M}(\mathscr{A})$ for every $u \in F$. By Theorem 1 of [3], there is some $G \in W_{p}$ such that $K=S^{-1}(G)$ is dense in $l_{0}^{\infty}(X, \mathscr{A})$ and barrelled.

Again by Theorems 1 and 14 of [8], the restriction of $S$ to $K$ admits a continuous extension $U$ in $l^{\infty}(X, \mathscr{A})$ with values in $G\left(\mathscr{T}_{G}\right)$, so $\mu$ is strongly additive because of Rosenthal's theorem [2]. Now $\mu$ is countably additive, since if $\left\{A_{n}, n=1,2, \ldots\right\}$ is a sequence of pairwise disjoint subsets of $X$ belonging to $\mathscr{A}$, we have that $\mu\left(\bigcup\left\{A_{n}, n=1,2, \ldots\right\}\right)$ is the only adherent point of the sequence $\left\{\sum\left(\mu\left(A_{p}\right), p=1,2, \ldots, n\right), n=1,2, \ldots\right\}$. In fact, $u \mu\left(\bigcup\left\{A_{n}, n=1,2, \ldots\right\}\right)=\left(\sum\left(u \mu\left(A_{p}\right), p=1,2, \ldots\right)\right.$, for each $u \in H$.

In the last theorem, the subspaces $L$ belonging to $W_{p}$ do not contain a copy of $l^{\infty}$ and are $\Gamma_{r}$ with a topology stronger than the induced one. Now we are going to prove that the former theorem can also be established if these subspaces $L$, provided with a topology stronger than the initial one, are of the class $\Lambda_{r}$ defined by Valdivia in [6].

We shall need the following well-known result of measure theory.

(c) Let $\left\{\lambda_{n}, n=1,2, \ldots\right\}$ be a sequence of elements of $\mathbf{M}(\mathscr{A})$. If $\lim \lambda_{n}(A)$ $=\lambda(A)$ for each $A \in \mathscr{A}$, then $\lambda \in \mathbf{M}(\mathscr{A})$.

This result states that $\mathbf{M}(\mathscr{A})\left(\sigma\left(\mathbf{M}(\mathscr{A}), l_{0}^{\infty}(X, \mathscr{A})\right)\right)$ is sequentially complete. The next proposition shows that $\mathbf{M}(\mathscr{A})(\sigma(\mathbf{M}(\mathscr{A}), E))$ is also sequentially complete when $E$ is a dense and barrelled subspace of $l_{0}^{\infty}(X, \mathscr{A})$. If $\mathscr{A}$ is infinite, there are in $\mathbf{M}(\mathscr{A})\left(\sigma\left(\mathbf{M}(\mathscr{A}), l_{0}^{\infty}(X, \mathscr{A})\right)\right)$ bounded sequences without adherent point in $\mathbf{M}(\mathscr{A})$. In fact, let $\left\{A_{n}, n \in \mathbb{N}\right\}$ be a sequence of nonempty pairwise disjoint elements of $\mathscr{A}$. Let $t_{n}$ be a point of $A_{n}$, and let $\delta_{n}$ be the Dirac measure on $t_{n}$. If $\lambda \in \mathbf{M}(\mathscr{A})$, we can find a $p$ such that if $M:=\bigcup\left\{A_{n}, n \geq p\right\}$, then $|\lambda(M)|<1 / 2$, and therefore, for $n \geq p$ we have that $\left|\left(\delta_{n}-\lambda\right)\right| \geq \delta_{n}(M)-|\lambda(M)|>1 / 2$. In [4], is shown that in $l^{1}\left(\sigma\left(l^{1}, l^{\infty}\right)\right)$ there are bounded sequences without any adherent point.

Proposition 1. If $E$ is a dense and barrelled subspace of $l_{0}^{\infty}(X, \mathscr{A})$ then the space $\mathbf{M}(\mathscr{A})(\sigma(\mathbf{M}(\mathscr{A}), E))$ is sequentially complete and $l_{0}^{\infty}(X, \mathscr{A})$ is contained in the bounded closure of $E$ with respect to the dual pair $(E, \mathbf{M}(\mathscr{A}))$.

Proof. The $E$-bounded subsets of $\mathbf{M}(\mathscr{A})$ are $E$-equicontinuous, and since $E$ is dense in $l_{0}^{\infty}(X, \mathscr{A})$, they are also $l_{0}^{\infty}(X, \mathscr{A})$-equicontinuous. Hence the $E$-bounded subsets of $\mathbf{M}(\mathscr{A})$ are $l_{0}^{\infty}(X, \mathscr{A})$-bounded. Thus, the second affirmation follows.

Now let $D$ be an absolutely convex subset of $\mathbf{M}(\mathscr{A})$ that is bounded and closed under $\sigma(\mathbf{M}(\mathscr{A}), E)$. Since $D$ is $\sigma\left(\mathbf{M}(\mathscr{A}), l_{0}^{\infty}(X, \mathscr{A})\right)$-compact, we have that the topologies coincide in $D$ and also the translations invariant uniformities induced by $\sigma\left(\mathbf{M}(\mathscr{A}), l_{0}^{\infty}(X, \mathscr{A})\right)$ and $\sigma(\mathbf{M}(\mathscr{A}), E)$.

Hence any $\sigma(\mathbf{M}(\mathscr{A}), E)$-Cauchy sequence $\left\{\lambda_{n}, n=1,2, \ldots\right\}$ in $\mathbf{M}(\mathscr{A})$ is also $\sigma\left(\mathbf{M}(\mathscr{A}), l_{0}^{\infty}(X, \mathscr{A})\right)$-Cauchy, and by result (c) there is some $\lambda \in \mathbf{M}(\mathscr{A})$ such that $\lim \lambda_{n}=\lambda$ under $\sigma\left(\mathbf{M}(\mathscr{A}), l_{0}^{\infty}(X, \mathscr{A})\right)$.

In particular, when $X=N$ and $\mathscr{A}=2^{N}$, we have that if $E$ is any dense and barrelled subspace of $l_{0}^{\infty}$ then $l^{1}\left(\sigma\left(l^{1}, E\right)\right)$ is weakly sequentially complete.

Proposition 2. Suppose that $W$ is a p-net contained in a space $F$, and let $f$ be a linear mapping from $l_{0}^{\infty}(X, \mathscr{A})$ into $F$ having closed graph in the product 
$l_{0}^{\infty}(X, \mathscr{A})\left(\sigma\left(l_{0}^{\infty}(X, \mathscr{A}), \mathbf{M}(\mathscr{A})\right)\right) \times F$. If each $L \in W_{p}$ has a locally convex topology $\mathscr{T}_{L}$ stronger than the induced by $F$ such that $L\left(\mathscr{T}_{L}\right)$ is a $\Lambda_{r}$-space, then there is some $G \in W_{p}$ containing the range of $f$ such that $f$ is weakly continuous with respect to the dual pairs $\left(l_{0}^{\infty}(X, \mathscr{A}), M(\mathscr{A})\right)$ and $\left(G, G\left(\mathscr{T}_{G}\right)^{\prime}\right)$. Proof. By Theorem 1 of [3] and Proposition 1, there is some $G$ such that $E:=f^{-1}(G)$ is dual locally complete with respect to the dual pair $(E, \mathbf{M}(\mathscr{A}))$, and its bounded closure $\check{E}$ contains $l_{0}^{\infty}(X, \mathscr{A})$.

Let $g$ be the restriction of $f$ to $E$. As $g$ has closed graph in the product $E(\sigma(E, \mathbf{M}(\mathscr{A}))) \times G\left(\mathscr{T}_{G}\right)$, then by [6, Theorems 2 and 6] the mapping $g$ has a continuous extension $h$ from $l_{0}^{\infty}(X, \mathscr{A})\left(\sigma\left(l_{0}^{\infty}(X, \mathscr{A}), \mathbf{M}(\mathscr{A})\right)\right)$ with values in $G\left(\sigma\left(G, G\left(\mathscr{T}_{G}\right)^{\prime}\right)\right)$.

The mappings $f$ and $g$ are continuous, taking in $F$ a locally convex topology weaker than the initial one, and both coincide in $E$. This fact concludes the proof.

Theorem 3. Let $\mu$ be a countably additive measure on $\mathscr{A}$ with values in a space $E$. Suppose that $F$ is a space with a p-net $W$ such that each $L \in W_{p}$ has a locally convex topology $\mathscr{T}_{L}$ stronger than the induced by $F$ under which $L\left(\mathscr{T}_{L}\right)$ is a $\Lambda_{r}$-space. Suppose finally that $f$ is a linear mapping from $E$ into $F$ with closed graph. Then there exists a $G \in W_{p}$ such that $f_{\mu}$ is a $G\left(\mathscr{T}_{G}\right)$-valued countably additive measure.

Proof. The mapping $S: l_{0}^{\infty}(X, \mathscr{A})\left(\sigma\left(l_{0}^{\infty}(X, \mathscr{A}), \mathbf{M}(\mathscr{A})\right)\right) \rightarrow E\left(\sigma\left(E, E^{\prime}\right)\right)$, such that $S(e(A))=\mu(A)$ for every $A \in \mathscr{A}$ is continuous, since $u \mu \in \mathbf{M}(\mathscr{A})$ for every $u \in E^{\prime}$.

Then $T=f S$ has closed graph in $l_{0}^{\infty}(X, \mathscr{A})\left(\sigma\left(l_{0}^{\infty}(X, \mathscr{A}), \mathbf{M}(\mathscr{A})\right)\right) \times F$.

By Proposition 2, there is some $G \in W_{p}$ such that $T\left(l_{0}^{\infty}(X, \mathscr{A})\right) \subset G$ and $T: l_{0}^{\infty}(X, \mathscr{A})\left(\sigma\left(l_{0}^{\infty}(X, \mathscr{A}), \mathbf{M}(\mathscr{A})\right)\right) \rightarrow G\left(\sigma\left(G, G\left(\mathscr{T}_{g}\right)^{\prime}\right)\right)$ is continuous.

If $v \in G\left(\mathscr{T}_{G}\right)^{\prime}$, then the continuity of $T$ implies that $v f \mu \in \mathbf{M}(\mathscr{A})$, and consequently the Orlicz-Pettis theorem implies that $f \mu$ is $\mathscr{T}_{G}$-countably additive.

Corollary. Let $\mu$ be an additive measure on $\mathscr{A}$ with values in a space $F$, and let $H$ be a $\sigma\left(F^{\prime}, F\right)$ total subset of $F^{\prime}$. Suppose that $F$ has a p-net $W$ such that each $L \in W_{p}$ has a locally convex topology $\mathscr{T}_{L}$ stronger than that induced by $F$, under which $L\left(\mathscr{T}_{L}\right)$ is a $\Lambda_{r}$-space. We also suppose that $u \mu$ is countably additive for every $u \in H$. Then there exists $a G \in W_{p}$ such that $\mu$ is a $G\left(\mathscr{T}_{G}\right)$-valued countably additive measure.

Proof. The corollary follows directly from Theorem 3 and Orlicz-Pettis theorem taking $E=F(\sigma(F,\langle H\rangle))$ and $f$ the identity map on $E$.

\section{ACKNOWLEDGMENT}

We express our gratitude to the referee for his valuable comments.

\section{REFERENCES}

1. J. Diestel, and B. Faires, On vector measures, Trans. Amer. Math. Soc. 198 (1974), 253-271.

2. L. Drewnowski, An extension of a theorem of Rosenthal on operators acting from $l^{\infty}(\Gamma)$, Studia Math. 57 (1976), 209-215.

3. J. C. Ferrando, and M. López-Pellicer, Strong barrelledness properties in $l_{0}^{\infty}(X, \mathscr{A})$ and bounded finite additive measures. Math. Ann. 287 (1990), 727-736. 
4. M. Levin, and S. A. Saxon, A note in the inheritance of properties of locally convex spaces by subspaces of countable codimension, Proc. Amer. Math. Soc. 29 (1971), 97-102.

5. P. Pérez Carreras, and J. Bonet Barrelled locally convex spaces, North Holland Math. Studies, vol. 131, Amsterdam, New York, Oxford, 1987.

6. M. Valdivia, On certain barrelled normed spaces. Ann. Inst. Fourier (Grenoble) 29 (1979), 39-56.

7. __ Mackey convergence and the closed graph theorem Arch. Math. 25 (1974), 649-656.

8. ___ Sobre el teorema de la gráfica cerrada. Collect. Math. 22 (1971), 51-72.

Departmento de Matemática Aplicada, Apartado 22012, 46071 Valencia, Spain 